\title{
THE MODULE INDEX AND INVERTIBLE IDEALS
}

\author{
BY \\ DAVID W. BALLEW( ${ }^{(1)}$
}

\begin{abstract}
A. Fröhlich used the module index to classify the projective modules of an order in a finite dimensional commutative separable algebra over the quotient field of a Dedekind domain. This paper extends Fröhlich's results and classifies the invertible ideals of an order in a noncommutatives eparable algebra. Several properties of invertible ideals are considered, and examples are given.
\end{abstract}

1. Introduction. Let $A$ be a Dedekind domain with quotient field $K$; let $\Sigma$ be a finite dimensional separable $K$-algebra. A subring $\Lambda$ of $\Sigma$ is said to be an $A$-order if $\Lambda$ contains $A, \Lambda$ is $A$-torsion free and $\Lambda$ contains a $K$-basis of $\Sigma$. There is always a maximal $A$-order $\Gamma$ containing $\Lambda$. A finite dimensional torsion free $A$-module is called an $A$-lattice.

Let $X$ be a finite dimensional torsion $A$-module. The order ideal, $\operatorname{ord}_{A} X$, is the product of the $A$-annihilators of the composition factors of an $A$-composition series of $X$. Let $M$ and $N$ be two $A$-lattices which span the same vector space over $K$; i.e., $K \otimes_{A} M \cong K \otimes_{A} N$. Define the module index of $M$ and $N$, [M:N], by $[M: N]=\left(\operatorname{ord}_{A} M / L\right) \cdot\left(\operatorname{ord}_{A} N / L\right)^{-1}$ where $L$ is any $A$-lattice such that $K \otimes_{A} L$ $=K \otimes_{A} M$ and $L \subseteq M \cap N$.

In [5], A. Fröhlich gives criteria in terms of the module index to decide the projectivity of $\Lambda$-modules when $\Sigma$ is commutative. More precisely, he shows that if $M$ is a $\Lambda$-module such that $K \otimes_{A} M \cong \Sigma^{(r)}$, then $M$ is $\Lambda$-projective if and only if $[\Gamma M: M]=[\Gamma: \Lambda]^{r}$, where $\Gamma$ is a maximal order over $\Lambda$ and $\Gamma M$ denotes the smallest $\Gamma$ module containing $M$. The object of this paper is to give analogues to Fröhlich's theorem when $\Sigma$ is noncommutative. We will give examples, however, to show that in the fairly simple case of a finite dimensional matrix algebra over the quotient field of a discrete rank one valuation ring, no direct extensions of either the necessity or the sufficiency of Fröhlich's theorem can be given.

It is well known that for integral domains, an ideal is invertible if and only if it is projective. However, the statement that projectivity implies invertibility is not necessarily true for noncommutative rings. Nevertheless, since invertibility is a

Presented to the Society, January 25, 1970; received by the editors May 9, 1969 and, in revised form, September 9, 1969.

AMS Subject Classifications. Primary, 1620; Secondary, 1648.

Key Words and Phrases. Orders, module index, invertible ideals, equivalent idempotents, separable algebra, reduced orders.

( $\left.{ }^{1}\right)$ Portions of this paper were included in the author's Ph.D. dissertation at the University of Illinois supervised by Robert Fossum.

Copyright (C) 1970, American Mathematical Society 
property of ideals that passes from the local case to the global and vice versa, we consider it in terms of Fröhlich type index conditions. We find that if the single index condition of Fröhlich's theorem is replaced by several index conditions in terms of idempotents and a multiplication condition on a two-sided full ideal, then we have necessary and sufficient conditions to insure the invertibility of the ideal. Further, it is not hard to see that for the case of full two-sided ideals and commutative $K$-algebras, these results imply Fröhlich's theorem.

2. Preliminaries. We first list a few basic properties of the module index. The proofs of these results follow directly from the definitions. Let $M, N, R$ be $A$ lattices spanning the same $K$-space.

1. $[M: N]_{p}=\left[M_{p}: N_{p}\right]$ for every prime ideal $p$ of $A$.

2. If $M \supseteq N$, then $[M: N] \subseteq A$.

3. $[M: N][N: R]=[M: R]$.

4. If $M=M_{1} \oplus M_{2}, N=N_{1} \oplus N_{2}$ with $M_{1}$ (resp. $M_{2}$ ) spanning the same space as $N_{1}$ (resp. $N_{2}$ ), then $\left[M_{1} \oplus M_{2}: N_{1} \oplus N_{2}\right]=\left[M_{1}: N_{1}\right]\left[M_{2}: N_{2}\right]$.

5. Let $f: v \rightarrow v^{\prime}$ be a $K$-isomorphism of finite dimensional vector spaces $v, v^{\prime}$. Let $M, N$ be two $A$-lattices spanning $v$. Then $[M: N]=[f(M): f(N)]$.

We will say that an ideal $I$ of $\Lambda$ is full if $I \otimes_{A} K \cong \Lambda \otimes_{A} K$.

We now let $A$ denote a complete discrete rank one valuation ring with quotient field $K$. Let $\pi$ be a generator of the maximal ideal of $A$, and let $\Sigma$ be a finite dimensional separable $K$-algebra.

Let $\Lambda$ denote an $A$-order in $\Sigma$, and let $\bar{\Lambda}=\Lambda / \pi \Lambda$. Then $\bar{\Lambda}$ is an algebra over the field $A /(\pi)$. Let $\overline{1}=\bar{e}_{1}+\bar{e}_{2}+\cdots+\bar{e}_{r}$ be a decomposition of $\bar{l}$ into primitive orthogonal idempotents. Then since $A$ is a complete discrete valuation ring, there are primitive orthogonal idempotents $e_{1}, \ldots, e_{r}$ in $\Lambda$ which map to $\bar{e}_{1}, \bar{e}_{2}, \ldots, \bar{e}_{r}$ by the natural map $\Lambda \rightarrow \bar{\Lambda}$ and such that $1=e_{1}+e_{2}+\cdots+e_{r}$.

We say that primitive orthogonal idempotents $e_{i}$ and $e_{j}$ in $\Lambda$ are equivalent in $\Lambda$ if $\left(e_{i} \Lambda e_{j}\right)\left(e_{j} \Lambda e_{i}\right)=e_{i} \Lambda e_{i}$. We will write $e_{i} \sim e_{j}$ to denote this equivalence.

Before we show that this is an equivalence relation, we will prove the following two lemmas.

LEMMA 1. Let $e_{i}$ and $e_{j}$ be primitive orthogonal idempotents in $\Lambda$. Then $e_{i} \sim e_{j}$ if and only if there are elements $x_{i j}$ in $e_{i} \Lambda e_{j}$ and $x_{j i}$ in $e_{j} \Lambda e_{i}$ such that $x_{i j} x_{j i}=e_{i}$ and $x_{j i} x_{i j}=e_{j}$.

Proof. Assume that $e_{i}$ is equivalent to $e_{j}$ in $\Lambda$, and let $x_{i j}$ in $e_{i} \Lambda e_{j}$ and $x_{j i}$ in $e_{j} \Lambda e_{i}$ be such that $x_{i j} x_{j i}=e_{i}$. Then, $x_{j i} x_{i j} x_{j i} x_{i j}=x_{j i} e_{i} x_{i j}=x_{j i} x_{i j}$; so $x_{j i} x_{i j}$ is idempotent in $e_{j} \Lambda e_{j}$. Since $e_{j}$ is a primitive idempotent, $x_{j i} x_{i j}$ is either zero or $e_{j}$. Because $x_{i j} x_{j i} x_{i j}=x_{i j} \neq 0, x_{j i} x_{i j}=e_{j}$.

The converse is clear. Q.E.D.

LEMMA 2. Let $e_{i}$ and $e_{j}$ be primitive orthogonal idempotents in $\Lambda$. Then $e_{i} \sim e_{j}$ if and only if $e_{i} \Lambda \cong e_{j} \Lambda$ (as right $\Lambda$-modules) if and only if $\Lambda e_{i} \cong \Lambda e_{j}$ (as left $\Lambda$-modules). 
Proof. Let $\sigma: e_{i} \Lambda \rightarrow e_{j} \Lambda, \tau: e_{j} \Lambda \rightarrow e_{i} \Lambda$ be inverse $\Lambda$-isomorphisms. Then $\sigma\left(e_{i}\right)$ $=e_{j} x$ for $x$ in $\Lambda$, so $\sigma\left(e_{i}^{2}\right)=\sigma\left(e_{i}\right) e_{i}=e_{j} x e_{i}$. Thus, we can take $x$ in $e_{j} \Lambda e_{i}$. Likewise, we have $\tau\left(e_{j}\right)=e_{i} y$ for $y$ in $e_{i} \Lambda e_{j}$. Now $e_{i}=\tau\left(\sigma\left(e_{i}\right)\right)=\tau\left(e_{j} x\right)=\tau\left(e_{j}\right) x=e_{i} y x, e_{j}=$ $\sigma\left(\tau\left(e_{j}\right)\right)=e_{j} x y$. But $e_{i} y=y, e_{j} x=x$, so $y x=e_{i}, x y=e_{j}$. Therefore, by Lemma 1 , $e_{i} \sim e_{j}$.

If $e_{i} \sim e_{j}$, then by Lemma 1 , there are elements $x$ in $e_{j} \Lambda e_{i}, y$ in $e_{i} \Lambda e_{j}$ such that $y x=e_{i}, x y=e_{j}$. Let $\sigma\left(e_{i}\right)=e_{j} x$, define a $\Lambda$-map from $e_{i} \Lambda$ to $e_{j} \Lambda$. Let $e_{j} \lambda$ be in $e_{j} \Lambda$. Then, $\sigma\left(e_{i} y\right)=e_{j} x y \lambda=e_{j} \lambda$, so $\sigma$ is onto. If $\sigma\left(e_{i} \lambda\right)=0$, then $x \lambda=0$ in $\Lambda$, so $y x \lambda=\lambda=0$. Hence $\sigma$ is an isomorphism.

The proofs for left modules are similar. Q.E.D.

Now it follows from Lemma 2 that " $\sim$ " is an equivalence relation.

LEMMA 3. If $e_{i}, e_{i}^{\prime}, e_{j}$ and $e_{j}^{\prime}$ are idempotents in $\Lambda$ such that $e_{i} \sim e_{i}^{\prime}$ and $e_{j} \sim e_{j}^{\prime}$, then $e_{i} \Lambda e_{j} \cong e_{i}^{\prime} \Lambda e_{j}^{\prime}$ (as A-modules).

Proof. First, assume that $e_{i}$ is equivalent to $e_{i}^{\prime}$. Then there is an $x_{i}$ in $e_{i} \Lambda e_{i}^{\prime}$ and $y_{i}$ in $e_{i}^{\prime} \Lambda e_{i}$ such that $x_{i} y_{i}=e_{i}$ and $y_{i} x_{i}=e_{i}^{\prime}$. Define $\eta: e_{i} \Lambda e_{j} \rightarrow e_{i}^{\prime} \Lambda e_{j}$ by $\eta\left(e_{i} z e_{j}\right)$ $=y_{i} z e_{j}$. If $y_{i} z e_{j}=0$, then $x_{i} y_{i} x e_{j}=e_{i} z e_{j}=0$. So $\eta$ is one-to-one. For $e_{i}^{\prime} w e_{j}$ in $e_{i}^{\prime} \Lambda e_{j}$, $\eta\left(e_{i} x_{i} w e_{j}\right)=y_{i} x_{i} w e_{j}=e_{i}^{\prime} w e_{j}$, so $\eta$ is onto. Thus $\eta$ is an isomorphism.

The proof for the case where $e$, is equivalent to $e_{j}^{\prime}$ is symmetric. Q.E.D.

An $A$-order $\Lambda$ in $\Sigma$ is said to be reduced if its identity has a decomposition into primitive orthogonal idempotents $1=e_{1}+\cdots+e_{n}$, such that no distinct $e_{i}$ and $e_{j}$ are equivalent [6, Appendix].

For the $A$-order $\Lambda$ in $\Sigma$, let $f_{1}, \ldots, f_{k}$ denote representatives of the distinct equivalence classes of equivalent idempotents. Let $f=f_{1}+\cdots+f_{k}$. Let $\tilde{\Lambda}=$ $f \Lambda f$; then $\tilde{\Lambda}$ is reduced in $f \Sigma f$.

LEMMA 4. The map $I \rightarrow \tilde{I}=f I f=I \cap \tilde{\Lambda}$ of two-sided $\Lambda$-modules to two-sided $\tilde{\Lambda}$-modules is one-to-one and preserves products if they are defined. Further, the map preserves sums and intersections.

Proof. Let $I$ and $J$ denote two-sided $\Lambda$-modules. The proofs that $(f I f) \cap(f J f)$ $=f(I \cap J) f$ and that $f I f+f J f=f(I+J) f$ are ordinary set inclusion arguments and are omitted.

We note that $\Lambda f \Lambda=\Lambda$. For, if $e_{i}$ is one of the idempotents of $\Lambda$, there is an $f_{j}$ such that $e_{i}$ is equivalent to $f_{j}$; i.e., $e_{i} \Lambda e_{i}=\left(e_{i} \Lambda f_{j}\right)\left(f_{j} \Lambda e_{i}\right)$. Hence, $e_{i}=e_{i} x f_{j} y e_{i}$ for elements $x$ and $y$ in $\Lambda$. Thus, in particular, $e_{i}$ is in $\Lambda f \Lambda$, so $1=\sum_{i=1}^{r} e_{i}$ is in $\Lambda f \Lambda$. Therefore, $\Lambda f \Lambda=\Lambda$.

Since $f I J f=f I \Lambda J f=f I \Lambda f \Lambda J f=(f I f)(f J f)$, it is clear that products are preserved when defined.

Finally, if $f I f=f J f$, then $\Lambda f \Lambda I \Lambda f \Lambda=\Lambda f \Lambda J \Lambda f \Lambda$. So, $\Lambda I \Lambda=I=J=\Lambda J \Lambda$. Hence, the $\operatorname{map} I \rightarrow \tilde{I}$ is one-to-one. Q.E.D.

We will say that a two-sided (fractional) ideal $I$ of $\Lambda$ is invertible if there is a two-sided $\Lambda$-module $J$ such that $I J=J I=\Lambda$. 
3. Index conditions in reduced orders. In this section, let $A$ be a complete discrete rank one valuation ring, let $\Lambda$ be a reduced $A$-order in $\Sigma$, and let $\Gamma$ be a maximal $A$-order containing $\Lambda$. Let $f_{1}, \ldots, f_{k}$ denote the nonequivalent primitive orthogonal idempotents of $\Lambda$, and assume they are primitive in $\Gamma$.

THEOREM 5. Let I be an invertible two-sided ideal in reduced A-order $\Lambda$. Then I is $\Lambda$-cyclic, $\Lambda$-free, and there is a permutation $\sigma$ of the set $\{1,2, \ldots, k\}$ such that for all $i$ and $j, i, j=1,2, \ldots, k$,

(a) $\left[f_{i} \Gamma f_{j}: f_{i} \Lambda f_{j}\right]=\left[f_{\sigma^{-1}(i)} I \Gamma f_{j}: f_{\sigma^{-1}(i)} I f_{j}\right]$

(b) $\left[f_{i} \Gamma f_{j}: f_{i} \Lambda f_{j}\right]=\left[f_{i} \Gamma I f_{\sigma(j)}: f_{i} I f_{\sigma(j)}\right]$,

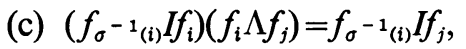

(d) $\left(f_{j} \Lambda f_{i}\right)\left(f_{i} I f_{\sigma(i)}\right)=f_{j} I f_{\sigma(i)}$.

Further, $[\Gamma: \Lambda]=[I \Gamma: I]=[\Gamma I: I]$.

Proof. Since $I$ is invertible, there is a two-sided $\Lambda$-module $J$ such that $I J=J I=\Lambda$. Thus

$$
f_{i} \Lambda f_{j}=\sum_{s=1}^{k}\left(f_{i} I f_{s}\right)\left(f_{s} J f_{j}\right)
$$

In particular,

$$
f_{i} \Lambda f_{i}=\sum_{s=1}^{k}\left(f_{i} I f_{s}\right)\left(f_{s} J f_{i}\right)
$$

Since $f_{i}$ is a primitive idempotent, $f_{i} \Lambda f_{i}$ has a single maximal ideal $M_{i}$, and the factor algebra $f_{i} \Lambda f_{i} / M_{i}$ is a division ring. Further, since $f_{i} \Lambda f_{i}$ is an $A$-algebra, every ideal is regular, so the Jacobson radical of $f_{i} \Lambda f_{i}$, being the intersection of all maximal right (or left) regular ideals, must be $M_{i}$ [7]. Consider

$$
f_{i} \Lambda f_{i} / M_{i}=\sum_{s=1}^{k}\left(f_{i} I f_{s}\right)\left(f_{s} J f_{i}\right) / M_{i}
$$

Then, since not all of the products $\left(f_{i} I f_{s}\right)\left(f_{s} J f_{i}\right)$ are in $M_{i}$ and since $f_{i} \Lambda f_{i} / M_{i}$ has no nontrivial ideals, there is an $s=\sigma(i), \sigma$ a function of $\{1,2, \ldots, k\}$ into itself, such that

$$
f_{i} \Lambda f_{i}=\left(f_{i} I f_{\sigma(i)}\right)\left(f_{\sigma(i)} J f_{i}\right)+M_{i}
$$

Then by [9, Theorem 4.1, p. 12],

$$
\left(f_{i} I f_{\sigma(i)}\right)\left(f_{\sigma(i)} J f_{i}\right)=f_{i} \Lambda f_{i} .
$$

Therefore, there is an $x_{i}$ in $f_{i} I f_{\sigma(i)}$ and a $y_{i}$ in $f_{\sigma(i)} J f_{i}$ such that $x_{i} y_{i}=f_{i}$. Since $y_{i} x_{i} y_{i} x_{i}$ $=y_{i} f_{i} x_{i}=y_{i} x_{i}$ is in $f_{\sigma(i)} \Lambda f_{\sigma(i)}$, and since $f_{\sigma(i)}$ is a primitive idempotent, then $y_{i} x_{i}$ is either zero or $f_{\sigma(i)}$. But $x_{i} y_{i} x_{i}=x_{i} \neq 0$, so $y_{i} x_{i}=f_{\sigma(i)}$. Such $x_{i}$ and $y_{i}$ can be found for all $i, i=1,2, \ldots, k$.

We claim that $\sigma$ is a permutation. Consider

$$
x_{1} \Lambda+\cdots+x_{k} \Lambda \subseteq I
$$


The left-hand side of (3) contains all of the $x_{i}$, so

$$
\left(x_{1} \Lambda+\cdots+x_{k} \Lambda\right) J=\Lambda,
$$

since the left-hand side of this expression contains all of the $x_{i} y_{i}$; therefore, it contains 1 . Hence, multiplying equation (4) on the right by $I$, we have

$$
I=\left(x_{1} \Lambda+\cdots+x_{k} \Lambda\right) J I,
$$

or

$$
I=x_{1} \Lambda+\cdots+x_{k} \Lambda
$$

Similarly,

$$
J=\Lambda y_{1}+\cdots+\Lambda y_{k} .
$$

On noting that $y_{j} x_{i}=0$ if $i \neq j$, we have that

$$
\Lambda=J I=\sum_{j=1}^{k} \Lambda y_{j} x_{j} \Lambda=\sum_{j=1}^{k} \Lambda f_{\sigma(j)} \Lambda .
$$

Now, if $f_{\alpha}$ is a member of the set $\left\{f_{1}, \ldots, f_{k}\right\}$, consider the equation

$$
f_{\alpha} \Lambda f_{\alpha}=\sum_{j=1}^{k}\left(f_{\alpha} \Lambda f_{\sigma(j)}\right)\left(f_{\sigma(j)} \Lambda f_{\alpha}\right)
$$

As before, there must be a $\beta$ in $\{1,2, \ldots, k\}$ such that

$$
f_{\alpha} \Lambda f_{\alpha}=\left(f_{\alpha} \Lambda f_{\sigma(\beta)}\right)\left(f_{\sigma(\beta)} \Lambda f_{\alpha}\right)
$$

But equation (7) implies that $f_{\alpha} \sim f_{\sigma(\beta)}$ which implies $\alpha=\sigma(\beta)$.

Thus, since $\sigma$ is a permutation, and $x_{i} y_{j}=0$ for $i \neq j,\left(x_{1}+\cdots+x_{k}\right) \Lambda J$ has all of the elements $x_{i} y_{i}$, and so it has 1 . Hence,

$$
\left(x_{1}+\cdots+x_{k}\right) \Lambda J=\Lambda \text {. }
$$

Multiplying equation (8) on the right by $I$, we have

$$
I=\left(x_{1}+\cdots+x_{k}\right) \Lambda J I=\left(x_{1}+\cdots+x_{k}\right) \Lambda,
$$

so $I$ is cyclic as a right module.

Since

$$
I=\sum_{i, j=1}^{k}\left(x_{1}+\cdots+x_{k}\right) f_{i} \Lambda f_{j}
$$

then

$$
f_{\sigma^{-1}(\alpha)} I f_{\beta}=x_{\sigma^{-1}(\alpha)} f_{\alpha} \Lambda f_{\beta}
$$

and so

$$
\begin{aligned}
\left(f_{\left.\sigma^{-1}{ }_{(i)} I f_{i}\right)\left(f_{i} \Lambda f_{j}\right)}=x_{\sigma^{-1}(i)}\left(f_{i} \Lambda f_{i}\right)\left(f_{i} \Lambda f_{j}\right)\right. \\
=x_{\sigma^{-1}{ }_{(i)} f_{i} \Lambda f_{j}} \\
=f_{\sigma^{-1}{ }_{(i)} I f_{j}}
\end{aligned}
$$

which establishes (c).

We claim that $I$ is isomorphic to $\Lambda$ and is therefore free. Set $x=x_{1}+\cdots+x_{k}$. Define $\eta: \Lambda \rightarrow I$ by $\eta(\lambda)=x . I J=J I=\Lambda$, so there is a $y$ in $J$ such that $x y=y x=1$; 
hence $x$ is a unit in $\Sigma$, and $\eta$ is one-to-one. $\eta$ is obviously onto, so it is an isomorphism.

By methods symmetric to the preceding, we have

$$
I=\Lambda\left(x_{1}+\cdots+x_{k}\right)=\Lambda x, \quad J=\left(y_{1}+\cdots+y_{k}\right) \Lambda .
$$

Therefore,

$$
\begin{aligned}
& I \Gamma=\left(x_{1}+\cdots+x_{k}\right) \Gamma=x \Gamma, \quad \Gamma I=\Gamma\left(x_{1}+\cdots+x_{k}\right)=\Gamma x, \\
& \Gamma J=\Gamma\left(y_{1}+\cdots+y_{k}\right), \quad J \Gamma=\left(y_{1}+\cdots+y_{k}\right) \Gamma .
\end{aligned}
$$

Since $x$ is a unit in $\Sigma$, we have

$$
[\Gamma: \Lambda]=[x \Gamma: x \Lambda]=[I \Gamma: I] \text {, and }[\Gamma: \Lambda]=[\Gamma x: \Lambda x]=[\Gamma I: I] \text {. }
$$

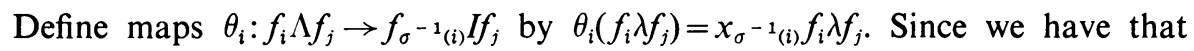

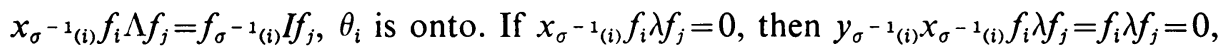
so the $\theta_{i}$ are one-to-one and are isomorphisms. The $\theta_{i}$ extend to isomorphisms

$$
\theta_{i}^{\prime}: f_{i} \Gamma f_{j} \rightarrow f_{\sigma^{-1}{ }_{(i)}} I \Gamma f_{j}
$$

by $\theta_{i}^{\prime}\left(f_{i} \gamma f_{j}\right)=x_{\sigma^{-1}(i)} f_{i} \gamma f_{j}$. Hence, for all $i$ and $j, i, j=1, \ldots, k$,

$$
\left[f_{i} \Gamma f_{j}: f_{i} \Lambda f_{j}\right]=\left[f_{\sigma^{-1}(i)} I \Gamma f_{j}: f_{\sigma^{-1}(i)} I f_{j}\right] .
$$

In order to obtain the other index conditions, we proceed symmetrically. Thus we show that

$$
\left(f_{i} \Lambda f_{i}\right)\left(f_{i} I f_{\sigma(i)}\right)=f_{j} I f_{\sigma(i)},
$$

and show that the maps $\mu_{i}: f_{i} \Lambda f_{j} \rightarrow f_{i} I f_{\sigma(j)}$ defined by $\mu_{i}\left(f_{i} \lambda f_{j}\right)=f_{i} \lambda f_{j} x_{\sigma(j)}$ are isomorphisms which extend to isomorphisms $\mu_{i}^{\prime}: f_{i} \Gamma f_{j} \rightarrow f_{i} \Gamma I f_{\sigma(j)}$. From this, the index condition (b) is immediate. Q.E.D.

In Example 14 we shall see that $[\tilde{\Gamma}: \tilde{\Lambda}]=[\tilde{\Gamma} \tilde{I}: \tilde{I}]=[\tilde{I} \tilde{\Gamma}: \tilde{I}]$ may be true in $\Lambda$ but that $[\Gamma: \Lambda] \neq[\Gamma I: I]$ and $[\Gamma: \Lambda] \neq[I \Gamma: I]$ in $\Lambda$. Further, this example will show that if $\Lambda$ is not reduced, then an invertible $\Lambda$-ideal $I$ may not be a cyclic $\Lambda$-ideal. Thus the condition that $\Lambda$ is reduced is essential in Theorem 5 .

Now we will prove the converse to Theorem 5. Define

$$
(\Lambda: I)_{r}=\{x \in \Sigma: I x \subseteq \Lambda\}, \quad(\Lambda: I)_{l}=\{x \in \Sigma: x I \subseteq \Lambda\} .
$$

THEOREM 6. Let I be a full two-sided ideal of the reduced A-order $\Lambda$ in $\Sigma$. Let $f_{1}, \ldots, f_{k}$ be the nonequivalent primitive orthogonal idempotents of $\Lambda$. If there is a permutation $\tau$ of the set $\{1,2, \ldots, k\}$ such that

(a) $\left[f_{i} \Gamma f_{i}: f_{i} \Lambda f_{i}\right]=\left[f_{\tau(i)} I \Gamma f_{i}: f_{\tau(i)} I f_{i}\right]$,

(b) $\left[f_{\tau(i)} \Gamma f_{\tau(i)}: f_{\tau(i)} \Lambda f_{\tau(i)}\right]=\left[f_{\tau(i)} \Gamma I f_{i}: f_{\tau(i)} I f_{i}\right]$,

(c) $\left(f_{\tau(i)} I f_{i}\right)\left(f_{i} \Lambda f_{j}\right)=f_{\tau(i)} I f_{j}$,

(d) $\left(f_{j} \Lambda f_{i}\right)\left(f_{i} I f_{\tau}{ }^{-1}(i)\right)=f_{j} I f_{\tau}{ }^{-1}(i)$,

for all $i$ and $j, i, j=1,2, \ldots, k$, then $I$ is invertible by the two-sided $\Lambda$-module $J=(\Lambda: I)_{r}=(\Lambda: I)_{l}$.

Proof. We consider $f_{\tau(i)} I f_{i}$ as a right $f_{i} \Lambda f_{i}$-module and $f_{\tau(i)} I \Gamma f_{i}$ as a right $f_{i} \Gamma f_{i^{-}}$ module. 
Since $A$ is a complete discrete rank one valuation ring and since $f_{i} \Gamma f_{i}$ has no idempotents other than $f_{i}$ or 0 , we can apply [14, Theorem 77.12, p. 548] and [1, Proposition 2.7, p. 8] to see that $f_{\tau(i)} I \Gamma f_{i}$ is a free $\Gamma$-module of rank 1 . Since $f_{i}$ is a primitive idempotent, $f_{i} \Gamma f_{i}$ has a single maximal ideal $N_{i}$ which must be the Jacobson radical of $f_{i} \Gamma f_{i}$. Further, $f_{i} \Gamma f_{i} / N_{i}$ is a division algebra over $A /(\pi)$.

We claim that $f_{\tau(i)} I \Gamma f_{i} / f_{\tau(i)} I \Gamma f_{i} N_{i}$ is a free $f_{i} \Gamma f_{i} / N_{i}$-module of rank one. For, if $\bar{x}_{1}, \ldots, \bar{x}_{r}$ is a basis of $f_{\tau(i)} I \Gamma f_{i} / f_{\tau(i)} I \Gamma f_{i} N_{i}$ over $f_{i} \Gamma f_{i} / N_{i}$ with preimages $x_{1}, \ldots, x_{r}$ in $f_{\tau(i)} I \Gamma f_{i}$ (by the natural map $f_{\tau(i)} I \Gamma f_{i} \rightarrow f_{\tau(i)} I \Gamma f_{i} / f_{\tau(i)} I \Gamma f_{i} N_{i}$ ), then let $B$ be the $f_{i} \Gamma f_{i}$ submodule generated by the set $\left\{x_{1}, \ldots, x_{r}\right\}$. Then $B+f_{\tau(i)} I \Gamma f_{i} N_{i}=f_{\tau(i)} I \Gamma f_{i}$. Hence, by Nakayama's Lemma, $B=f_{\tau(i)} I \Gamma f_{i}$, and $r=1$. Thus

$$
\left(f_{\tau(i)} I \Gamma f_{i} / f_{\tau(i)} I \Gamma f_{i} N_{i}: f_{i} \Gamma f_{i} / N_{i}\right)=1 .
$$

Consider the composed map

$$
\theta_{i}: f_{\tau(i)} I f_{i} \rightarrow f_{\tau(i)} I \Gamma f_{i} \rightarrow f_{\tau(i)} I \Gamma f_{i} / f_{\tau(i)} I \Gamma f_{i} N_{i} .
$$

Let $z_{i}$ be an element of $f_{\tau(i)} I f_{i}$ which does not lie in a kernel of $\theta_{i}$. The images from $f_{\tau(i)} I f_{i}$ generate $f_{\tau(i)} I \Gamma f_{i} / f_{\tau(i)} I \Gamma f_{i} N_{i}$ over $f_{i} \Gamma f_{i} / N_{i}$, and so $z_{i}$ freely generates $f_{\tau(i)} I \Gamma f_{i} / f_{\tau(i)} I \Gamma f_{i} N_{i}$ over $f_{i} \Gamma f_{i} / N_{i}$. Hence

$$
z_{i}+f_{\tau(i)} I \Gamma f_{i} N_{i}=f_{\tau(i)} I \Gamma f_{i}
$$

and by Nakayama's Lemma,

$$
z_{i} f_{i} \Gamma f_{i}=f_{\tau(i)} I \Gamma f_{i} .
$$

Therefore, we have shown that $f_{\tau(i)} I \Gamma f_{i}$ is freely generated over $f_{i} \Gamma f_{i}$ by an element $z_{i}$ of $f_{\tau(i)} I f_{i}$. Certainly, we can find such $z_{i}$ for all $i$.

From the sequence

$$
f_{i} \Gamma f_{i} \cong z_{i} f_{i} \Gamma f_{i}=f_{\tau(i)} I \Gamma f_{i} \supseteq f_{\tau(i)} I f_{i} \supseteq z_{i} f_{i} \Lambda f_{i} \cong f_{i} \Lambda f_{i}
$$

and by hypothesis (a), we have that

for all $i, i=1, \ldots, k$.

$$
f_{\tau(i)} I f_{i}=z_{i} f_{i} \Lambda f_{i}
$$

Set $z=z_{1}+\cdots+z_{k}$, and we will show that $z \Lambda=I$. Note that $\left(f_{i} \Lambda f_{i}\right)\left(f_{i} \Lambda f_{j}\right)=f_{i} \Lambda f_{j}$ for all $i$ and $j$. Thus,

$$
\begin{aligned}
z \Lambda & =\sum_{i=1}^{k} z_{i} \Lambda=\sum_{i, j} z_{i} \Lambda f_{j}=\sum_{i, j} z_{i} f_{i} \Lambda f_{j}=\sum_{i, j} z_{i}\left(f_{i} \Lambda f_{i}\right)\left(f_{i} \lambda f_{j}\right) \\
& =\sum_{i, j}\left(f_{\tau(i)} I f_{i}\right)\left(f_{i} \Lambda f_{j}\right)=\sum_{i, j} f_{\tau(i)} I f_{j}=I
\end{aligned}
$$

(using hypothesis (c) and the fact that $\tau$ is a permutation).

Now we consider $f_{\tau(i)} I f_{i}$ as a left $f_{\tau(i)} \Lambda f_{\tau(i)}$-module and $f_{\tau(i)} \Gamma I f_{i}$ as a left $f_{\tau(i)} \Gamma f_{\tau(i)^{-}}$ module. Hence, by a proof symmetric to the above, we can pick an element $z_{i}^{\prime}$ in $f_{\tau(i)} I f_{i}$ which does not lie in the kernel of the composed map

$$
\theta_{i}^{1}: f_{\tau(i)} I f_{i} \rightarrow f_{\tau(i)} \Gamma I f_{i} \rightarrow f_{\tau(i)} \Gamma I f_{i} / N_{\tau(i)} f_{\tau(i)} \Gamma I f_{i} .
$$


We claim that $z_{i}$ can be chosen to be $z_{i}^{\prime}$. Certainly, this is true if $f_{\tau(i)} I f_{i}$ is not contained in the union of $f_{\tau(i)} I \Gamma f_{i} N_{i}$ and $N_{\tau(i)} f_{\tau(i)} \Gamma I f_{i}$ (considered as subsets of $\Gamma$ ). Assume that $f_{\tau(i)} I f_{i}$ is contained in this union. It is known that $z_{i}$ is not in $f_{\tau(i)} I \Gamma f_{i} N_{i}$, so it must be in $N_{\tau(i)} f_{\tau(i)} \Gamma I f_{i}$. Also, $z_{i}^{\prime}$ is not in $N_{\tau(i)} f_{\tau(i)} \Gamma I f_{i}$, so it must be in $f_{\tau(i)} I \Gamma f_{i} N_{i}$. Consider $z_{i}+z_{i}^{\prime}$ in $f_{\tau(i)} I f_{i}$. If $z_{i}+z_{i}^{\prime}$ is in $f_{\tau(i)} I \Gamma f_{i} N_{i}$, then $z_{i}$ must be in $f_{\tau(i)} I \Gamma f_{i} N_{i}$, a contradiction. Assuming $z_{i}+z_{i}^{\prime}$ is in $N_{\tau(i)} f_{\tau(i)} \Gamma I f_{i}$ implies that $z_{i}^{\prime}$ is in $N_{\tau(i)} f_{\tau(i)} \Gamma I f_{i}$, again a contradiction. Therefore, $f_{\tau(i)} I f_{i}$ is not contained in the above union.

Hence, by proofs completely symmetric to the previous ones,

$$
f_{\tau(i)} I f_{i}=f_{\tau(i)} \Lambda f_{\tau(i)} z_{i}
$$

(using index condition (b)), and $\Lambda z=I$ (using condition (d)).

Since $I$ is full, $z \Sigma=\Sigma$ and $\Sigma z=\Sigma$. Thus, let $w$ be in $\Sigma$ such that $z w=1=w z$. Set $J=\Lambda w \Lambda$. Then

$$
J I=\Lambda w \Lambda z \Lambda=\Lambda w z \Lambda=\Lambda, \text { and } \quad I J=\Lambda z \Lambda w \Lambda=\Lambda z w \Lambda=\Lambda .
$$

Thus $I$ is invertible.

Since $J \subseteq(\Lambda: I)_{r}$ and $(\Lambda: I)_{r}=J I(\Lambda: I)_{r} \subseteq J \Lambda=J$, we have that $J=(\Lambda: I)_{r}$. Similarly, $J=(\Lambda: I)_{l}$. Q.E.D.

It is appropriate to note here that in the commutative case every order is reduced, so Theorems 5 and 6 generalize Fröhlich's Theorem to the noncommutative case.

THEOREM 7. Let I be a full two-sided ideal in the reduced $A$-order $\Lambda$. Let $f_{1}, \ldots, f_{k}$ be a set of reduced idempotents for $\Lambda$. Then I is $\Lambda$-invertible if and only if the following conditions are satisfied:

(a) $\left[f_{i} \Gamma I^{k} f_{i}: f_{i} I^{k} f_{i}\right]=\left[f_{i} \Gamma I^{2 k} f_{i}: f_{i} I^{2 k} f_{i}\right]$,

(b) $\left[f_{i} I^{k} \Gamma f_{i}: f_{i} I^{k} f_{i}\right]=\left[f_{i} I^{2 k} \Gamma f_{i}: f_{i} I^{2 k} f_{i}\right]$,

(c) $\left[f_{i} \Lambda f_{i}: f_{i} I^{2 k} f_{i}\right]=\left[f_{i} \Lambda f_{i}: f_{i} I^{k} f_{i}\right]^{2}$,

(d) $\left(f_{i} I^{k} f_{i}\right)\left(f_{i} \Lambda f_{j}\right)=f_{i} I^{k} f_{j}$,

(e) $\left(f_{j} \Lambda f_{i}\right)\left(f_{i} I^{k} f_{i}\right)=f_{j} I^{k} f_{i}$.

Proof. Assume that $I$ is $\Lambda$-invertible. In Theorem 5, we showed that there was a permutation $\sigma$ of $1,2, \ldots, k$ and that there were elements $x_{i}$ in $f_{i} I f_{\sigma(i)}$ such that

$$
x_{\sigma^{-1}(i)} f_{i} \Lambda f_{j}=f_{\sigma^{-1}(i)} I f_{j},
$$

and such that for $x=x_{1}+\cdots+x_{k}$, we have $\Lambda x=x \Lambda=I$. Now,

$$
\begin{aligned}
& x^{2}=\left(x_{1}+\cdots+x_{k}\right)^{2}=\sum_{i=1}^{k} x_{i} x_{\sigma(i)}, \\
& x^{3}=\left(x_{1}+\cdots+x_{k}\right)^{3}=\sum_{i=1}^{k} x_{i} x_{\sigma(i)} x_{\sigma^{2}(i)}, \\
& x^{n}=\left(x_{1}+\cdots+x_{k}\right)^{n}=\sum_{i=1}^{k} x_{i} x_{\sigma(i)} \cdots x_{\sigma^{n}(i)} .
\end{aligned}
$$


So since $\sigma^{k}=1, x^{k}=z_{1}+\cdots+z_{k}$ with $z_{i}$ an element of $f_{i} I^{k} f_{i}$. Set $z=z_{1}+\cdots+z_{k}$. Then $I^{k}=z \Lambda=\Lambda z$. Hence we have

$$
I^{k}=\left(z_{1}+\cdots+z_{k}\right) \Lambda=\left(z_{1} f_{1} \Lambda+\cdots+z_{k} f_{k} \Lambda\right) .
$$

So $f_{i} I^{k} f_{i}=z_{i} f_{i} \Lambda f_{i}$. Similarly, $f_{i} I^{k} f_{i}=f_{\mathfrak{i}} \Lambda f_{i} z_{i}$. We note further that

$$
\begin{aligned}
z_{i} f_{i} I^{k} f_{i} & =z_{i} f_{i} \Lambda f_{i} f_{i} I^{k} f_{i} \\
& =z_{i} f_{i} \Lambda f_{i} z_{i} f_{i} \Lambda f_{i} \\
& =z_{i} f_{i} \Lambda f_{i} .
\end{aligned}
$$

Since we have $f_{i} I^{2 k} f_{i}=z_{i}^{2} f_{i} \Lambda f_{i}=f_{i} \Lambda f_{i} z_{i}^{2}$, then $f_{i} I^{k} f_{i}$ is $\Lambda$-isomorphic to $f_{i} I^{2 k} f_{i}$ by an isomorphism which extends to an isomorphism from $f_{i} I^{k} \Gamma f_{i}$ to $f_{i} I^{2 k} \Gamma f_{i}$. Thus the index condition (b) holds. By similar arguments, it is clear that the index condition (a) holds.

Now,

$$
\begin{aligned}
{\left[f_{i} \Lambda f_{i}: f_{i} I^{2 k} f_{i}\right] } & =\left[f_{i} \Lambda f_{i}: f_{i} I^{k} f_{i}\right]\left[f_{i} I^{k} f_{i}: f_{i} I^{2 k} f_{i}\right] \\
& =\left[f_{i} \Lambda f_{i}: f_{i} I^{k} f_{i}\right]\left[f_{i} \Lambda f_{i} z_{i}: f_{i} \Lambda f_{i} z_{i}^{2}\right] \\
& =\left[f_{i} \Lambda f_{i}: f_{i} I^{f_{i}}\right]\left[f_{i} \Lambda f_{i}: f_{i} \Lambda f_{i} z_{i}\right] \\
& =\left[f_{i} \Lambda f_{i}: f_{i} I^{k} f_{i}\right]\left[f_{i} \Lambda f_{i}: f_{i} I^{k} f_{i}\right] \\
& =\left[f_{i} \Lambda f_{i}: f_{i} I^{k} f_{i}\right]^{2} .
\end{aligned}
$$

Hence index condition (c) is satified.

Finally,

$$
\begin{aligned}
\left(f_{\mathfrak{i}} I^{k} f_{\mathfrak{i}}\right)\left(f_{\mathfrak{i}} \Lambda f_{\mathfrak{i}}\right) & =z_{\mathfrak{i}} f_{\mathfrak{i}} \Lambda f_{\mathfrak{i}} f_{\mathfrak{i}} \Lambda f_{\mathfrak{i}} \\
& =z_{\mathfrak{i}} f_{i} \Lambda f_{\mathfrak{i}} \\
& =z_{\mathfrak{i}} I^{k} f_{\mathfrak{i}} .
\end{aligned}
$$

So the condition (d) is true, and by similar arguments condition (e) is true.

Now we will assume the conditions (a) through (e). In Theorem 6, we saw that we could find elements $z_{i}$ in $f_{i} I^{k} f_{i}$ which freely generate $f_{i} I^{k} \Gamma f_{i}$ over $f_{i} \Gamma f_{i}$ as right $f_{i} \Gamma f_{i}$-modules and which freely generate $f_{i} \Gamma I^{k} f_{i}$ over $f_{i} \Gamma f_{i}$ as left $f_{i} \Gamma f_{i}$-modules.

Then

$$
\begin{aligned}
{\left[f_{i} \Lambda f_{i}: f_{i} I^{2 k} f_{i}\right] } & =\left[f_{i} \Lambda f_{i}: f_{i} I^{k} f_{i}\right]\left[f_{i} I^{k} f_{i}: f_{i} I^{2 k} f_{i}\right] \\
& =\left[f_{i} \Lambda f_{i}: f_{i}{ }^{k} f_{i}\right]\left[f_{i} \Gamma I^{k} f_{i}: f_{i} \Gamma I^{2 k} f_{i}\right] \\
& =\left[f_{i} \Lambda f_{i}: f_{i} I^{k} f_{i}\right]\left[f_{i} \Gamma f_{i} z_{i}: f_{i} \Gamma f_{i} z_{i}\right] \\
& =\left[f_{i} \Lambda f_{i}: f_{i} I^{k} f_{i}\right]\left[f_{i} \Gamma f_{i}: f_{i} \Gamma f_{i_{i}} z_{i}\right] \\
& =\left[f_{i} \Lambda f_{i}: f_{i} I^{k} f_{i}\right]\left[f_{i} \Gamma f_{i}: f_{i} \Gamma I^{k} f_{i}\right]
\end{aligned}
$$

since conditions (c) hold for all $i$. We must have

or

$$
\left[f_{i} \Lambda f_{i}: f_{i} I^{k} f_{i}\right]=\left[f_{i} \Gamma f_{i}: f_{i} \Gamma I^{k} f_{i}\right],
$$

$$
\left[f_{i} \Gamma f_{i}: f_{i} \Lambda f_{i}\right]=\left[f_{i} \Gamma I^{k} f_{i}: f_{i} I^{k} f_{i}\right] .
$$


In a similar fashion we have

$$
\left[f_{i} \Gamma f_{i}: f_{i} \Lambda f_{i}\right]=\left[f_{i} I^{k} \Gamma f_{i}: f_{i} I^{k} f_{i}\right] .
$$

Thus in view of conditions (d) and (e), we have from Theorem 6 that $I^{k}$ is $\Lambda$ invertible, i.e., $I$ is $\Lambda$-invertible. Q.E.D.

4. Reduction from a Dedekind domain. In this section, we will let $D$ be a Dedekind domain with quotient field $K$. Assume $\Sigma$ is a finite dimensional separable $K$-algebra, $\Lambda$ a $D$-order in $\Sigma$ and $\Gamma$ a maximal $D$-order in $\Sigma$ containing $\Lambda$. Let $P$ be a maximal prime ideal in $D$. Let $K_{(P)}$ denote the completion of $K$ at $P$, and let $D_{(P)}$ be the complete discrete valuation ring in $K_{(P)}$. Let $\Sigma_{(P)}=\Sigma \otimes_{D} D_{(P)}, \Lambda_{(P)}$ $=\Lambda \otimes_{D} D_{(P)}, I_{(P)}=I \otimes_{D} D_{(P)}$, etc. There are well-known canonical embeddings of $\Gamma_{(P)}, \Lambda_{(P)}, I_{(P)}$ into $\Sigma_{(P)}$ [5]. For the $D$-order $\Lambda$ and a maximal prime ideal $P$ of $D$, we will let $e_{1}, e_{2}, \ldots, e_{r_{P}}$ be the primitive orthogonal idempotents of $\Lambda_{(P)}$ and assume that they are primitive in $\Gamma_{(P)}$. Let $f_{1}, f_{2}, \ldots, f_{k_{P}}$ be representatives of the distinct equivalence classes of idempotents in $\Lambda_{(P)}, f=f_{1}+\cdots+f_{k_{P}}$ and let $\tilde{\Lambda}_{(P)}=f \Lambda_{(P)} f$, $\tilde{I}_{(P)}=f I_{(P)} f$ for $I$ a two-sided $\Lambda$-module.

We will now give "global" versions of the theorems of the preceding section.

THEOREM 8. Let I be an invertible two-sided ideal in the D-order $\Lambda$. Let $\{P\}$ denote the set of all maximal prime ideals of $D$. Then there are permutations $\sigma$ of the sets $\left\{1,2, \ldots, k_{P}\right\}$ such that for $i$ and $j$ and all primes $P$ of $D$,

(a) $\left[f_{i} \Gamma_{(P)} f_{j}: f_{i} \Lambda_{(P)} f_{j}\right]=\left[f_{\sigma^{-1}{ }_{(i)}} I \Gamma_{(P)} f_{j}: f_{\sigma^{-1}{ }_{(i)}} I_{(P)} f_{j}\right]$,

(b) $\left[f_{i} \Gamma_{(P)} f_{j}: f_{i} \Lambda_{(P)} f_{j}\right]=\left[f_{i} \Gamma I_{(P)} f_{\sigma(j)}: f_{i} I_{(P)} f_{\sigma(j)}\right]$,

(c) $\left(f_{\sigma}^{-1}{ }_{(i)} I_{(P)} f_{i}\right)\left(f_{i} \Lambda_{(P)} f_{j}\right)=f_{\sigma^{-1}{ }_{(i)}} I_{(P)} f_{j}$,

(d) $\left(f_{j} \Lambda_{(P)} f_{i}\right)\left(f_{i} I_{(P)} f_{\sigma(i)}\right)=f_{j} I_{(P)} f_{\sigma(i)}$,

where $f_{1}, \ldots, f_{k_{P}}$ are the reduced idempotents of $\tilde{\Lambda}_{(P)}$.

Proof. Since $D$ is a Dedekind domain, $I$ is reflexive and $I=\bigcap_{P} I_{(P)}$. Also, $I_{(P)}=\Lambda_{(P)}$ for almost all $P$. So if each $I_{(P)}$ is invertible by $J_{(P)}$ in $\Lambda_{(P)}$, set $J=\bigcap_{P} J_{(P)}$, and consider $I J .(I J)_{(P)}=\Lambda_{(P)}$ for all $P$, so $I J=\Lambda$ since $\Lambda=\bigcap_{P} \Lambda_{(P)}=I J$. Certainly if $I$ is invertible by $J$ in $\Lambda$, then $(I J)_{(P)}=I_{(P)} J_{(P)}=\Lambda_{(P)}$, so $I_{(P)}$ is invertible in $\Lambda_{(P)}$ for all $P$. Hence by [5, Theorem 2, p. 204], we need only prove the theorem for each $D_{(P)}$.

Let $I_{(P)}$ be a two-sided $\Lambda_{(P)}$-ideal which is invertible in $\Lambda_{(P)}$ by $J_{(P)}$. Setting $\tilde{I}_{(P)}=f I_{(P)} f$ and $\tilde{J}_{(P)}=f J_{(P)} f$, we have by Lemma 4 that $\tilde{I}_{(P)}$ is a two-sided $\tilde{\Lambda}_{(P)}$-ideal which is invertible in $\tilde{\Lambda}_{(P)}$ by $\tilde{J}_{(P)}\left(\tilde{I}_{(P)} \tilde{J}_{(P)}=\left(f I_{(P)} f\right)\left(f J_{(P)} f\right)=f I_{(P)} J_{(P)} f=f \Lambda_{(P)} f=\tilde{\Lambda}_{(P)}\right)$. Since $\tilde{\Lambda}_{(P)}$ is reduced, we can apply Theorem 5 to obtain a permutation $\sigma$ of the set $\left\{1,2, \ldots, k_{P}\right\}$ such that the conclusions (a), (b), (c) and (d) are true. Q.E.D.

THEOREM 9. If $I$ is a full two-sided ideal of the D-order $\Lambda$ such that for all $P$ there is a permutation $\sigma$ of the $\left\{1,2, \ldots, k_{P}\right\}$ having the properties

(a) $\left[f_{j} \Gamma_{\left(P_{\alpha}\right)} f_{j}: f_{j} \Lambda_{(P)} f_{j}\right]=\left[f_{\sigma^{-1}(j)} I \Gamma_{(P)} f_{j}: f_{\sigma}^{-1}{ }_{(j)} I_{\left(P_{\alpha}\right)} f_{j}\right]$, 
(b) $\left[f_{\sigma^{-1}{ }_{(j)}} \Gamma_{(P)} f_{\sigma^{-1}{ }_{(j)}:}: f_{\sigma^{-1}(j)} \Lambda_{(P)} f_{\sigma^{-1}(j)}\right]=\left[f_{\sigma^{-1}{ }_{(j)}} \Gamma I_{(P)} f_{j}: f_{\sigma^{-1}\left({ }^{(j)}\right)} I_{(P)} f_{j}\right]$,

(c) $\left(f_{\sigma^{-1}{ }_{(i)}} I_{(P)} f_{i}\right)\left(f_{i} \Lambda_{(P)} f_{j}\right)=f_{\sigma^{-1}(i)} I_{(P)} f_{j}$,

(d) $\left(f_{j} \Lambda_{(P)} f_{i}\right)\left(f_{i} I_{(P)} f_{\sigma(i)}\right)=\left(f_{j} I_{(P)} f_{\sigma(i)}\right)$,

for all $i$ and $j$, where $f_{1}, \ldots, f_{k_{P}}$ denote the reduced idempotents of $\tilde{\Lambda}_{(P)}$. Then $I$ is invertible in $\Lambda$.

Proof. Just as in the proof of Theorem 8, to prove that $I$ is invertible in $\Lambda$, it is sufficient to prove that for all $P, I_{(P)}$ is invertible in $\Lambda_{(P)}$. Further, in Theorem 8, we showed that if $I_{(P)}$ was $\Lambda_{(P)}$-invertible, then $\tilde{I}_{(P)}$ was $\tilde{\Lambda}_{(P)}$-invertible. Note that if $\tilde{I}_{(P)}$ is $\tilde{\Lambda}_{(P)}$-invertible by $\tilde{J}_{(P)}$, then

$$
\begin{aligned}
\tilde{\Lambda}_{(P)} & =\tilde{I}_{(P)} \tilde{J}_{(P)} \\
& =\left(f I_{(P)} f\right)\left(f J_{(P)} f\right) \\
& =f I_{(P)} f J_{(P)} f .
\end{aligned}
$$

Thus,

$$
\begin{aligned}
\tilde{\Lambda}_{(P)} & =f I_{(P)} \Lambda_{(P)} f \Lambda_{(P)} J_{(P)} f \\
& =f I_{(P)} \Lambda_{(P)} J_{(P)} f \\
& =f I_{(P)} J_{(P)} f .
\end{aligned}
$$

So $I_{(P)} J_{(P)}$ maps to $\tilde{\Lambda}_{(P)}$ in the map of $\Lambda_{(P)}$-modules defined in Lemma 4. Hence $I_{(P)} J_{(P)}=\Lambda_{(P)}$. Thus, $I_{(P)}$ is $\Lambda_{(P)}$-invertible if and only if $\tilde{I}_{(P)}$ is $\tilde{\Lambda}_{(P)}$-invertible. Therefore, it suffices to prove the result for reduced orders over complete discrete valuation rings, and by Theorem 6, the proof is completed. Q.E.D.

Finally we give the "global version" of Theorem 7.

Theorem 10. Let I be a full two-sided ideal in the D-order $\Lambda$. Let $P$ denote a maximal prime in $D$ and let $f_{1}, \ldots, f_{k_{P}}$ then I is $\Lambda$-invertible if and only if the following conditions hold for every maximal prime $P$ and for all $i$ and $j$ :

(a) $\left[f_{i} \Gamma I_{(P)}^{k_{P}} f_{i}: f_{i} I_{(P)}^{k_{P}} f_{i}\right]=\left[f_{i} \Gamma I_{(P)}^{2 k_{P}} f_{i}: f_{i} I_{(P)}^{2 k_{P}} f_{i}\right]$,

(b) $\left[f_{i} I^{k_{P}} \Gamma_{(P)} f_{i}: f_{i} I_{(P)}^{k_{P}} f_{i}\right]=\left[f_{i} I^{2 k_{P}} \Gamma_{(P)} f_{i}: f_{i} I_{(P)}^{2 k_{P}} f_{i}\right]$,

(c) $\left[f_{i} \Lambda_{(P)} f_{i}: f_{i} f_{(P)}^{2 k_{P}} f_{i}\right]=\left[f_{i} \Lambda_{(P)} f_{i}: f_{i} I_{(P)}^{k_{p}} f_{i}\right]^{2}$

(d) $\left(f_{i} I_{(P)}^{k_{P}} f_{i}\right)\left(f_{i} \Lambda_{(P)} f_{j}\right)=\left(f_{i} I_{(P)}^{k_{P}} f_{j}\right)$,

(e) $\left(f_{j} \Lambda_{(P)} f_{i}\right)\left(f_{i} I_{(P)}^{k_{p}} f_{i}\right)=\left(f_{j} I_{(P)}^{k_{P}} f_{i}\right)$.

Proof. t.t was proved in Theorems 8 and 9 that $I$ is $\Lambda$-invertible if and only if $I_{(P)}$ is $\Lambda_{(P)}$-invertible for every maximal prime $P$ in $D$, and $I_{(P)}$ is $\Lambda_{(P)}$-invertible if and only if $\tilde{I}_{(P)}$ is $\tilde{\Lambda}_{(P)}$-invertible. Hence this result follows from Theorem 7. Q.E.D.

5. Examples. This section will give examples and counterexamples relevant to the preceding sections. Let $A$ be a discrete valuation ring with quotient field $K$ and prime element $\pi$. Let $\Sigma_{n}$ be the $K$-algebra of $n \times n$ matrices. Let $\Lambda=\left[\pi^{r_{i}} A\right]$ denote an $A$-order in $\Sigma$. This notation means that the $(i, j)$ positions of $\Lambda$ has elements in the form $\pi^{r_{i j} a}$ with $r_{i j}$ an integer and $a$ any element of $A$. 
We note the following lemma.

Lemma 11. Let $\Lambda=\left[\pi^{r_{i j}} A\right], I=\left[\pi^{s_{i j}} A\right]$ and $(\Lambda: I)=\{x: I x \subseteq \Lambda\}=\left[\pi^{m_{i j}} A\right]$. Then $I$ is left $\Lambda$-projective if and only if for every $P, P=1, \ldots, n$, there is a $k_{P}, 1 \leqq k_{P} \leqq n$ such that $m_{P, k_{P}}=-s_{k_{P} P}$.

The lemma is actually a restatement in the language of matrices of a classical result of Cartan and Eilenberg [3, p. 132].

EXAMPLE 12. In $\Sigma_{2}$, the fact that a full left ideal is $\Lambda$-projective does not necessarily imply that $[\Gamma I: I]=[\Gamma: \Lambda]$. Note that this example shows that one part of Fröhlich's Theorem does not extend to all finite dimensional central simple algebras.

Set $\Gamma=\Lambda_{2}$, and let $\Lambda$ be the $A$-order contained in $\Gamma$ of the form

$$
\Lambda=\left[\begin{array}{cc}
A & \pi^{2} A \\
\pi A & A
\end{array}\right]
$$

Let $I$ be the left ideal of the form

$$
I=\left[\begin{array}{cc}
\pi^{4} A & \pi^{3} A \\
\pi^{2} A & \pi A
\end{array}\right]
$$

Then $\Gamma I$ has the form

$$
\Gamma I=\left[\begin{array}{ll}
\pi^{2} A & \pi A \\
\pi^{2} A & \pi A
\end{array}\right]
$$

Then $[\Gamma: \Lambda]=\pi^{3} A$ and $[\Gamma I: I]=\pi^{4} A$, and $[\Gamma I: I] \neq[\Gamma: \Lambda]$. Using Lemma 11 , it is easy to check that $I$ is left $\Lambda$-projective.

EXAMPLE 13. In $\Sigma_{2}$, the fact that $[\Gamma I: I]=[\Gamma: \Lambda]$ does not imply that a full left ideal is $\Lambda$-projective. Therefore, this example along with Example 11 shows that Fröhlich's Theorem has no direct extension to central simple algebras.

Set $\Gamma=\Lambda_{2}$ and let $\Lambda$ be as in Example 11. Let $I$ be the left $\Lambda$-ideal of the form

Then $\Gamma I$ is of the form

$$
\left[\begin{array}{cc}
\pi^{3} A & \pi^{3} A \\
\pi^{2} A & \pi A
\end{array}\right]
$$

$$
\left[\begin{array}{ll}
\pi^{2} A & \pi A \\
\pi^{2} A & \pi A
\end{array}\right]
$$

Then $[\Gamma: \Lambda]=\pi^{3} A=[\Gamma I: I]$. It is easy to check that $I$ is not $\Lambda$-projective by using Lemma 11.

One of the conclusions of Theorem 5 is that $[\Gamma: \Lambda]=[I \Gamma: I]=[\Gamma I: I]$ for $I$ an invertible two-sided $\Lambda$-ideal. Therefore it is reasonable to ask if this condition is strong enough to imply invertibility. The following example shows that the condition is not strong enough even if $I$ is assumed to be both right and left $\Lambda$ projective.

EXAMPLE 14. In $\Sigma_{2}$ there is a two-sided $\Lambda$-deal which is right and left projective and such that $[I \Gamma: I]=[\Gamma I: I]=[\Gamma: \Lambda]$ but is not invertible in $\Lambda$. 
Let

and

$$
\Gamma=\Lambda_{2}, \quad \Lambda=\left[\begin{array}{cc}
A & \pi A \\
\pi A & A
\end{array}\right]
$$

$$
I=\left[\begin{array}{cc}
\pi^{2} A & \pi A \\
\pi A & A
\end{array}\right]
$$

Then $I$ is a two-sided $\Lambda$-ideal and

$$
[\Gamma I: I]=[I \Gamma: I]=[\Gamma: \Lambda]=\pi^{2} A \text {. }
$$

It is seen by Lemma 11 and its symmetric counterpart for right ideals that $I$ is both right and left $\Lambda$-projective. $I$ is not $\Lambda$-invertible since it is not cyclic and $\Lambda$ is reduced.

EXAMPLE 15. This example shows that if $\Lambda$ is not a reduced $A$-order, then an invertible ideal $I$ may not be cyclic. Further, $[\Gamma I: I] \neq[\Gamma: \Lambda]$ and $[I \Gamma: I] \neq[\Gamma: \Lambda]$, but $[\tilde{I} \tilde{\Gamma}: \tilde{I}]=[\tilde{\Gamma}: \tilde{\Lambda}]=[\tilde{\Gamma} \tilde{I}: \tilde{I}]$. So, in particular, the condition that $\Lambda$ be a reduced $A$-order is essential in Theorem 5 .

Let

$$
\Lambda=\left[\begin{array}{ccc}
A & A & A \\
\pi A & A & \pi A \\
A & A & A
\end{array}\right]
$$

and

$$
I=\left[\begin{array}{ccc}
\pi A & A & \pi A \\
\pi A & \pi A & \pi A \\
\pi A & A & \pi A
\end{array}\right] .
$$

$\Lambda$ is not reduced since $e_{11} \sim e_{33} . I$ is $\Lambda$-invertible by

If $\Gamma=\Lambda_{3}$, then

$$
J=\left[\begin{array}{ccc}
A & \pi^{-1} A & A \\
A & A & A \\
A & \pi^{-1} A & A
\end{array}\right]
$$

$$
[\Gamma: \Lambda]=\pi^{2} A, \quad[\Gamma I: I]=\pi^{6} A, \quad[I \Gamma: I]=\pi^{3} A .
$$

To see that $I$ is not cyclic, assume the contrary. On noting that $I^{2}=\pi \Lambda$, we see that there are elements $a, b$ in $\Lambda$ such that $x^{2}=\pi a, x b x=\pi$. Then $(\operatorname{det} a)(\operatorname{det} b)=1$, and $(\operatorname{det} x)^{2}=\pi^{3}(\operatorname{det} b)$. Let det $x=\pi^{s}$. Then $2 s=3$, a contradiction.

Now set $f_{1}=e_{11}, f_{2}=e_{22}$, and $f=f_{1}+f_{2}$. Then

Set

$$
\tilde{\Lambda}=f \Lambda f=\left[\begin{array}{cc}
A & A \\
\pi A & A
\end{array}\right], \quad \tilde{I}=f I f=\left[\begin{array}{cc}
\pi A & A \\
\pi A & \pi A
\end{array}\right] .
$$

$$
x=\left[\begin{array}{ll}
0 & 1 \\
\pi & 0
\end{array}\right]
$$


Then, $\tilde{I}=\tilde{\Lambda} \tilde{x}=\tilde{x} \tilde{\Lambda}$, and $\Lambda=\Lambda_{2}$, so

$$
[\tilde{\Gamma}: \tilde{\Lambda}]=[\tilde{\Gamma} \tilde{I}: \tilde{I}]=[\tilde{I} \tilde{\Gamma}: \tilde{I}]=\pi A
$$

\section{REFERENCES}

1. M. Auslander and O. Goldman, Maximal orders, Trans. Amer. Math. Soc. 97 (1960), 1-24. MR 22 \#8034.

2. D. Ballew, The module index, projective modules and invertible ideals, Ph.D. Thesis, University of Illinois, Urbana, 1969.

3. H. Cartan and S. Eilenberg, Homological algebra, Princeton Univ. Press, Princeton, N. J., 1956. MR 17, 1040.

4. C. Curtis and I. Reiner, Representation theory of finite groups and associative algebras, Pure and Appl. Math., vol. 11, Interscience, New York, 1962. MR 26 \#2519.

5. A. Fröhlich, Invariants for modules over commutative separable orders, Quart. J. Math. Oxford Ser. (2) 16 (1965), 193-232. MR 35 \#1583.

6. R. Gordon, Rings faithfully represented on their left socle, J. Algebra 7 (1967), 303-342. MR 36 \#1483.

7. N. Jacobson, Structure of rings, rev. ed., Amer. Math. Soc. Colloq. Publ., vol. 37, Amer. Math. Soc., Providence, R. I., 1964. MR 36 \#5158.

8. J. Murtha, Hereditary orders over principal ideal domains, Ph.D. thesis, University of Wisconsin, Madison, 1964.

9. M. Nagata, Local rings, Interscience Tracts in Pure and Appl. Math., no. 13, Interscience, New York, 1962. MR 27 \#5790.

South Dakota School of Mines and Technology, Rapid City, South Dakota 